\title{
The Temporal Epistemic Anomaly
}

\author{
Peter Riggs \\ Australian National University \\ Research School of Physics and Engineering \\ Quantum Science \\ Building 38a Science Road, Canberra, Australian Capital \\ Territory 2601 \\ Australia \\ peter.riggs@anu.edu.au
}

\section{Article info}

CDD: 121

Received: 11.10.2018; Accepted: 22.10.2018

DOI: http://dx.doi.org/10.1590/0100-6045.2018.V41N3.PR

\section{Keywords:}

Temporal anomaly

Causal loop

Non-invented information

Backwards causation

Time travel

\begin{abstract}
It is not uncommon in time travel stories to find that the mechanism by which the time travel is achieved is not invented. A time traveller could journey to his/her own past and give the designs of the time travel machine to his/her earlier self as $\mathrm{s} /$ he was given the designs as a younger person. These designs never get thought up by anyone. Such a situation would conflict with the usual conception of the acquisition of knowledge. This situation is called the Temporal Epistemic Anomaly and would arise if knowledge is gained at a time prior to the information in question being transmitted but is not discovered or invented at any time. This article examines the implications of information propagating around a causal chain that is closed in time (which is required to create the Anomaly) and whether this information need have a specific origin point.
\end{abstract}




\section{THE TEMPORAL EPISTEMIC ANOMALY}

\section{INTRODUCTION}

Our 'Information Age' graphically displays that there are many ways in which (macroscopic) information may be found, extracted, created, stored, retrieved, transferred, merged, copied, sorted, manipulated, corrupted, and lost. The same information may exist in a variety of types including 'hardcopy', optical, chemical, and electromagnetic forms. Information can be exactly duplicated so that the original recording of an instance of information (its initial information carrier) may be obliterated and yet the information remain in existence. Nonetheless, what counts as 'information' depends somewhat on context although all instances of information have to have physical manifestations, i.e. information is contained in physical systems (Landauer 1996, p.188; Karnani et al. 2009, p.2157). No attempt will be made to define 'information' as this is beyond the scope of the current article (see the comprehensive discussions in: Lombardi 2004; Zins 2007; Karnani et al. 2009; Floridi 2011; and Mingers \& Standing 2018). Instead, 'information' will be taken as implicitly understood. What will be examined here are the odd implications of information being propagated around a causal chain of events that is closed in time (called a causal loop) and whether this scenario will stand up to scrutiny.

\section{INFORMATION AND THE TEMPORAL EPISTEMIC ANOMALY}

Let's begin by laying out the specifics of the topic. Consider the hypothetical situation where an instance of information is received and understood by someone (thereby constituting knowledge for our purposes) at a time 
before the information is transmitted (by whatever means) but is not discovered or invented at any point in time. The information must propagate completely around a causal loop in order for it not to be invented or discovered. (Note that although a causal loop is required here, such loops are not themselves necessitated by 'backwards' in time causal influences, cf. Riggs 1991; Monton 2009). The gaining of knowledge from information 'circulating' a causal loop conflicts with the standard conception of the acquisition of knowledge because nobody 'thinks up' or gains the knowledge by physically probing the world. This sort of scenario will be referred to as the Temporal Epistemic Anomaly. Other names used in the literature include "Knowledge Paradox", "Epistemological Paradox" and "Bootstrap Paradox", even though the depicted situation is not strictly paradoxical. However, we should at this point, dispel the very common objection that the (alleged) selfdefeating paradoxes of time travel demonstrate that sending messages to an earlier time (or indeed time travel to the past) is logically impossible and therefore that the Anomaly also is impossible. The contention that 'backwards' time travel is logically impossible has been shown elsewhere to be false (e.g. see: Horwich 1987; Smith 1997; Riggs 1997; Ismael 2003; Hanley 2004; Smeenk \& Wüttrich 2011; Kutach 2013; and Berkovitz 2017).

Although the Temporal Epistemic Anomaly could be achieved by sending some kind of modulated signal to the past, the complexity of the problem increases when information is conveyed by more tangible means, e.g. if a 'hardcopy' representation of the information is sent. This is best illustrated by the classic example of the non-invention of a time travel machine. A traveller journeys to his/her own past and presents the plans of the time travel machine used to make the journey to his/her earlier self, just as s/he was provided with as a younger person. The time travel machine's design information passes completely around a 
causal loop but there is no part of the loop (and therefore no particular point in space and time) where the design information is created.

Interest in the Temporal Epistemic Anomaly scenario has increased markedly in the last few decades for at least three reasons. First, there is an on-going fascination with the general public for all things time travel related. The popular appeal of time travel has led to a plethora of science fiction literature/ motion pictures/ television series involving travel to an earlier time and/or information transfer to the past. Motion pictures (with serious plots) in which time travel features prominently continue to appear every few years. Prime examples from the last ten years include: Stargate: Continuum (2008); Star Trek (2009); Looper (2012); Predestination (2013); Edge of Tomorrow (2014); Terminator Genisys (2015); Synchronicity (2015); Reset (2017); and The Paradox (2017).

A second reason is that several mathematical models which allow travel to an earlier time or the transmission of information to the past have been detailed in the technical literature (e.g. Morris et al. 1988; Friedman et al. 1990; Gott 1991; Visser 1996; Mallett 2003; Ralph \& Downes 2012; Yuan et al. 2015; Tippett \& Tsang 2017) along with popular physics books (e.g. Thorne 1994; Gott 2001; Mallett \& Henderson 2008; Kaku 2009; Clegg 2011; Al-Khalili 2016). These models have made the concept of time travel to the past 'respectable' for formal academic study. The third reason for increased interest is that the possibility of time travel has received attention in several recent worldwide television documentaries on current physics (e.g. BBCHorizon; History Channel).

The late American philosopher David Lewis provided the largely accepted response to the Temporal Epistemic Anomaly. He claimed that every event on a causal loop has an explanation for its happening, viz. every event is caused by other events on that loop. He wrote: 
Each event on the loop has a causal explanation, being caused by events elsewhere on the loop. This is not to say that the loop as a whole is caused or explicable ... The parts of the loop are explicable, the whole of it is not. Strange! But not impossible ... (Lewis 1976, pp.148-149).

Strange indeed! The whole loop is inexplicable but its parts are not, nevertheless the loop is not impossible. Should this really be considered a sound description?

Lewis thought that it is not possible to offer an explanation for the existence of causal loops and simply placed them alongside the greatest of all mysteries, e.g. the Big Bang (Lewis 1976, p.149). Nevertheless, one would expect that such mysteries are more fundamental than causal loops and therefore grouping them together does not provide sufficient justification for the claim that an explanation of causal loops is not possible. Further, Lewis's specification implies that a causal loop is isolated from its surroundings, i.e. events external to the loop have no causal connections to events forming the loop. This cannot be so for no (macroscopic) event, be it part of a causal loop or not, is totally isolated from its surrounding environment. Macroscopic events are unavoidably involved in causal interactions such as reflection/ refraction/ absorption/ defraction of electromagnetic radiation, heat gain or loss, gravitational attraction, etc. It has been adequately argued elsewhere that although events on a causal loop are dependent on other events on the loop, these same events will also be affected by and affect events which are not part of the loop (e.g. Riggs 1997, pp.59-60; Henley 2004, p.125). If events which are not on a causal loop are partial causes of the events comprising the loop, then it is not the case that the whole loop is itself uncaused. What Lewis should 
have said was that even though causal loops are not themselves inexplicable, the origin of information transmitted completely around such a loop is unexplained.

All portrayals of the Temporal Epistemic Anomaly that have appeared over the previous two decades are also less than satisfactory (individual examples include: Davies 2001, pp.113-116; Greene 2004, pp.450-451; Lockwood 2005, pp.174-177; Kaku 2005, p.142; Toomey 2007, pp.208-209; Dainton 2010, pp.136-138; Weinert 2013, pp.243-244; and Nahin 2017, pp.221-225). These treatments have merely restated the problem and/or accepted that the Anomaly exists if time travel to the past is possible. In doing so, they offered little or no analysis and have perpetuated some misconceptions. A typical approach is found in Brian Greene's popularisation of contemporary physics, The Fabric of the Cosmos:

Apparently, in a world that allows time travel both to the future and to the past, knowledge can materialize out of thin air. Although not quite as paradoxical as preventing your own birth, this is positively weird (Greene 2004, p.451, italics added).

A 2004 article by Richard Hanley did explore the relevant issues but only ended up concluding that "... the correct explanation [of the Anomaly] is, coincidence, on a massive scale" (Hanley 2004, p.142, italics in original). Surely a better explanation of the Anomaly can be uncovered! In order to achieve this, more thorough analysis is called for than has previously been attempted.

It should also be appreciated that a detailed analysis of the Temporal Epistemic Anomaly is of academic importance as such an analysis may serve to provide a check on the internal consistency of some physical theories (Lobo \& Crawford 2003, p.296). This is the case since 
relativity in concert with quantum mechanics does not exclude the possibility of time travel to the past (see: Deutsch \& Lockwood 1994; Earman et al. 2009; Lindley 2011; Ralph \& Downes 2012). Therefore, it would be both informative (in terms of better understanding knowledge acquisition) and useful (as a check on the consistency of physical theories) to determine whether there are logical and/or physical factors that would prevent the Anomaly.

Let's begin by defining 'normal circumstances' as those where there is no causation 'backwards' in time. In normal circumstances, it is always theoretically possible to isolate the origin of any instance of information. Of course, no one in their right mind would assert that the circumstances of causal loops are normal. In the context of causal loops, we wish to enquire whether it is necessary for information to have an origin in space and time. It seems intuitive that everything in the universe should have a beginning which can be accounted for in a manner that is both consistent and coherent. This intuition is supported (at least for macroscopic entities) by the following well-established conditions regarding the emergence of complex physical systems (e.g. forests, animals, books, digital media, etc.):

- Such systems do not spontaneously come into existence but are built up in stages over periods of time (or produced by technologies that were made in stages over periods of time);

- All processes of assembling such systems (by natural or artificial means) require the expenditure of energy; and

- The extra organisation in the universe created by producing such systems results in dis-organisation ('messiness') somewhere else, e.g. trees are cut down and pulped to make the paper needed for printing books. 
Implicit here is that macroscopic physical entities are not created ex-nibilo. If the above conditions could be shown not to apply to information and the laws of physics do not prohibit travel to an earlier time then there would appear to be no substantial reason to claim that the Temporal Epistemic Anomaly scenario is not possible.

\section{Physical Laws, EnTropy and Causal loops}

Consider the application of the laws of physics. If it could be demonstrated that, in principle, the existence of non-invented information on a causal loop would violate any law of physics then this would indicate that all information must have an origin in space and time. Would time travel to the past violate physical conservation laws (e.g. conservation of mass-energy, electric charge, etc.)? Apparently not! It has been shown that conservation laws still apply in General Relativistic models of the universe that allow time travel (Friedman et al. 1990; Lossev \& Novikov 1992; Kutach 2003; Earman et al. 2009). Since the issue of the non-violation of conservation laws has already been adequately dealt with, we need not discuss it further and the reader is referred to the cited references.

The Second Law of Thermodynamics is another matter altogether. The Second Law is not a conservation law but is the law of physics most relevant to information acquisition (see: Karnani et al. 2009; Floridi 2011). What's more, there are claims regularly made that the Second Law prevents any form of 'backwards' in time causation or time travel to the past (e.g. Popovic 2014; Taylor 2015). It is for these reasons that we shall contemplate (in some detail) the Second Law within the context of time travel and come to a conclusion regarding its impact on the Anomaly. A textbook statement of the Second Law is: 
In the neighbourhood ... of any equilibrium state of a [thermodynamic] system ... there exist states that cannot be reached (are inaccessible) by reversible adiabatic processes (Zemansky \& Dittman 1981, p.164, italics in original).

The state of a thermodynamic system is specified when its thermodynamic coordinates (e.g. temperature, volume, pressure) are detailed (Zemansky \& Dittman 1981, pp.3-4; Dugdale 1996, p.12; Stowe 2007, p.17). The Second Law applies if the state of the thermodynamic system is adiabatically isolated from its surrounding environment (Reif 1981, p.122; Dugdale 1996, pp.60-62). Adiabatic isolation is where a system can only be disturbed by mechanical means (e.g. vibration), i.e. the system cannot absorb heat from or emit heat to its surroundings.

The parameter called entropy is generally used in the description of thermodynamic states. Entropy refers to a physical system and not to the system's individual constituents (Morris 1985, p.122). Entropy is a measure of the number of accessible states of a system (Reif 1981, p.116; Stowe 2007, p.127). If the system is adiabatically isolated then there will be either no change in its entropy or the change will be positive. This is referred to as the law of the increase of entropy and is an alternative expression of the Second Law (Stowe 2007, p.128). Hans Reichenbach illustrated the Second Law with an example of a footprint left on a sandy beach. How is the formation of this footprint related to entropy? It turns out that the total entropy of both the footprint and the foot which made it would have increased (Reichenbach 1956, p.150). However, if we were only aware of the beach (and not that anyone had ever walked upon it) then we would infer that the creation of the footprint would result in a decrease in entropy (i.e. an apparent violation of the Second Law). 
Consider now the situation of a causal loop. Causation would appear to run 'in reverse' on part of a causal loop as judged against the vast majority of causal processes. Would this also entail a violation of the Second Law? Since one section of a causal loop has causation 'reversed', the prima facie reply to this question is that there would be a violation. Let's look at the issue more closely as this response has been advanced as showing time travel to the past to be physically impossible. Suppose we have a set of events, the burning of a piece of paper say. This piece of paper accompanies a time traveller who is moving along the 'backwards' section of a causal loop inside a time travel machine (as might be achieved by a spacecraft under very special circumstances). The piece of paper is lit by the traveller at the start of his/her voyage in time, corresponding to a time $t_{2}$ in a suitable frame of reference external to the time travel machine. The piece of paper is completely burnt at the completion of the time journey, corresponding to time $\mathrm{t}_{1}$, where $\mathrm{t}_{1}<\mathrm{t}_{2}$ in the external reference frame. The traveller sees the burning as a normal process in which entropy appears to have increased, i.e. what constituted the piece of paper has changed into smoke and ashes.

Suppose an observer in the external frame (a 'chronology-respecting' observer) views the same events. This could conceivably be done if the spaceship/ time machine has large windows so that images of the burning paper could be registered by a camera on another spaceship which is capable of recording for extended periods (centuries if necessary). The chronology-respecting observer would witness ashes and smoke at time $\mathrm{t}_{1}$, paper appearing from a receding flame during the interval $t_{1}$ to $t_{2}$ and a whole unlit piece of paper at time $t_{2}$. This observer seems to have seen a thermodynamic system evolve into an inaccessible state, just as if the observer had watched a video recording of a burning process in normal 
circumstances being run in reverse. It is to be expected that observers in different frames of reference will not agree about some details regarding events, but does not the chronology-respecting observer also witness a violation of the Second Law?

The time traveller observes processes consistent with the Second Law. Whilst engaged in time travelling, all events inside the time travel machine have a temporal sequence that appears normal to the time traveller. In other words, events occur as expected within the confines of an operating time travel machine including the ordinary processes of everyday life. However, the chronologyrespecting observer believes that there is a violation of the Second Law because s/he observes a process in which an inaccessible physical state appears to evolve spontaneously. Now there must either be a violation of the Second Law or not, which requires either the time traveller or the chronology-respecting observer to be mistaken. Although obviously related to a possible violation of the Second Law, the questions regarding who is mistaken and why constitute separate issues (which have not previously been satisfactorily addressed).

In order to answer the questions of whether there is a violation of the Second Law, who is mistaken in the above anecdote and why the mistake occurs, the following two points need to be acknowledged:

(a) The relevant change in total entropy includes changes in entropy in both the time travel machine and its immediate surroundings. What is essential to note is that the time travel machine is not adiabatically isolated from its surrounding environment. There will always be some minimal environmental interactions (e.g. reflection of light, loss of heat, etc.) as the time travel machine 
travels 'backwards' in time (Lossev \& Novikov 1992, p.2311); and

(b) The overall change in entropy (for the purposes of the Second Law) is gauged relative to the time evolution of the vast majority of physical processes (cf. Nikolić 2006, pp.261-262). This ensures consistency with the empirically verified form of the Second Law together with general experience.

The Second Law will not be violated if, in the external time interval beginning at $\mathrm{t}_{1}$ and ending at $\mathrm{t}_{2}$, the change in total entropy of the time travel machine and its immediate surroundings is positive. This could be determined by an entropy tally over the time interval $t_{1}$ to $t_{2}$ which included the changes in entropy of:

- the burnt paper;

- what the paper was in contact with (e.g. the air inside the time travel machine); and

- the surrounding region of space through which the time journey is undertaken but excluding other entropy generating sources. Just as with Reichenbach's sandy footprint, the total entropy determination must include the relevant interactions.

In the absence of an entropy tally, what justification do we have for claiming that the Second Law of Thermodynamics would hold in this scenario? Well, with the inclusion of the above points (a) and (b), it is the same justification used for entropy increasing in situations not involving time travel. Despite the Second Law being a statistical law, violations on macroscopic scales (i.e. involving enormous numbers of particles) do not occur, as Reichenbach has indicated: 
The law of the increase of entropy is guaranteed by the law of large numbers ... but is not of the type of the strict laws of physics ... which are regarded as exempt from possible exceptions (Reichenbach 1956, pp.54-55).

Reichenbach was not quite correct in the above statement for one more component is required. What guarantees the increase of entropy (relative to the vast majority of causal processes) is the statistics governing huge collections of particles together with the entropy of the universe having been much lower in the distant past. The proposition that the very early universe was in a state of extremely low entropy is called the Past Hypothesis (Albert 2000, p.96) and there are very convincing reasons for accepting it (see: Callender 2010; Patel \& Lineweaver 2017; Riggs 2018).

Given the above points (a) \& (b) and that the Past Hypothesis is correct, the (relevant) change in total entropy from $t_{1}$ to $t_{2}$ will be positive. This is sufficient to indicate that any contention that the Second Law must be violated if time travel to the past occurred cannot be legitimately upheld and, as a result, a supposed violation cannot be used as a reason to physically rule out the Temporal Epistemic Anomaly. Further, it then follows that the chronologyrespecting observer has been deceived due to his/her perspective of the time traveller's unique circumstances. 


\section{INFORMATION CARRIERS AND CAUSAL LOOPS}

In addition to the well established conditions stated at the end of Section 2, all carriers of information (e.g. books, radio signals) are subject to physical laws and processes. Consequently, an origin in space and time is required - all information carriers have to be created in a regular manner at some point in space and time. A single carrier of information cannot merely 'circulate' a causal loop, i.e. neither entering nor leaving the loop, for if this were the case then there would be no point of origin. Therefore, on a causal loop, there must be a minimum of two carriers which transfer information to each other. Clearly then, a time traveller cannot provide a set of plans of a time travel machine to his/her earlier self if it is the same set of plans as $\mathrm{s} /$ he was given as a younger person.

This applies more generally to any physical object. Several fictional time travel stories have taken a large degree of dramatic liberty in respect to objects not being created. Such circumstances have been referred to in the literature as 'object loops'. Fictional examples depicting 'object loops' include a pocket watch in the movie Somewhere in Time (1980) and a pair of antique eyeglasses in the movie Star Trek IV (1986). In these stories, the objects are not created but just exist on causal loops. Some of the logical difficulties with 'object loops' have been sufficiently dealt with (e.g. Hanley 2004, pp.131-135; Wasserman 2018, pp.158-164) and will not be addressed. Since an origin in space and time is mandated for all physical entities, it should be apparent that no single object can only exist entirely on a causal loop.

What about information on a causal loop? If a causal loop is not a closed system for the purposes of information transfer, then events other than ones constituting the loop may affect what is propagated around the loop, including the introduction of items of information. Events external 
to the loop might thereby be identified as the origin(s) of instances of information on the loop. Therefore, only information which remains 'entirely on the loop' without interference to its content (as distinct from its physical carriers) is involved in the Temporal Epistemic Anomaly. In the context of information we should replace the thermodynamic term 'adiabatically isolated' with the more relevant term 'content isolated'. Information being content isolated means that the information content cannot be interferred with.

Let's consider an example with two information carriers on a causal loop. Suppose someone external to the causal loop (a chronology-respecting observer) sees a physical representation of an instance of information (i.e. a carrier of this information - call it carrier (1) apparently appear 'from nowhere' at time $t_{A}$. Denote this occurrence as event A. The information carrier has travelled 'backwards' in time. This is why the carrier just appears at $t_{A}$ from the perspective of the chronology-respecting observer. Further suppose that, shortly after this time, the information contained in carrier (1) is copied to another information carrier (call it carrier (2) which did not initially hold any (macroscopic) information. Carrier (1) is then destroyed. Carrier (2) continues in existence and at time $t_{B}\left(>t_{A}\right)$ encounters the younger version of carrier (1) which holds no information at this time (call this event B). The information contained in carrier (2) is then copied to carrier (1). Carrier (2) is destroyed once the copying is completed and carrier (1) begins to travel 'backwards' in time. A causal loop for the transfer of information is formed from event $\mathrm{A}$ to event $\mathrm{B}$ and back again, as depicted in Figure 1. 


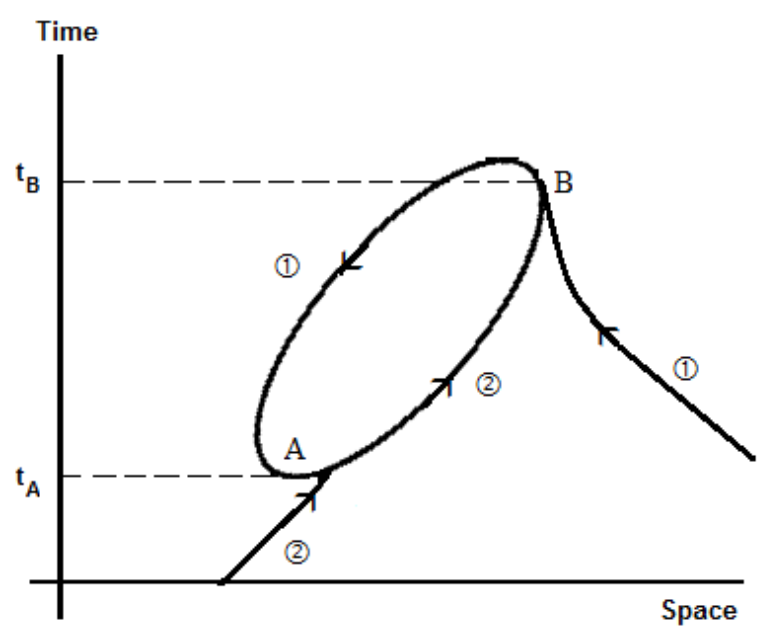

Figure 1: Causal Loop with Two Information Carriers

Such a scenario explains the existence of the information carriers but not the origin of the information itself if the information is content isolated.

Since information carriers are physical entities, there are always going to be thermodynamic and mechanical aspects applicable to the information representation, storage, and transfer (Karnani et al. 2009, p.2156). In particular, all structured systems undergo degradation over time so that any information content present may be altered or even destroyed. What's more, the larger the detail in an individual instance of information, the greater is the likelihood that its content will not be faithfully transferred when being copied, due to the following factors:

- Other data can be introduced which corrupts the original information content;

- The processes required to copy information from one carrier to another can result in the deletion of (all or part) of the information; and 
- The transfer of information (especially highly complicated data) can and regularly does lead to copying errors.

Such factors have previously been touted as reasons for why the Anomaly cannot occur (e.g. Toomey 2007, p.209). However, these sort of contingent practical factors cannot, by themselves, totally rule out the acquisition of non-invented information via a causal loop. The in-principle existence of non-invented information, given 'backwards' causation or time travel to the past (as the laws of physics do not prevent this), cannot be eliminated by purely practical considerations and remains an unexplained theoretical possibility.

If we leave time travel aside for a moment, it is important to realise that non-invented information may be produced in normal circumstances too. The production of non-invented information without transmission to the past is actually well known but is not usually taken seriously. This is attested to by the fact that it is occasionally enacted for comic entertainment value. The story of the chimpanzee placed in front of a personal computer by an unsuccessful writer comes to mind. The chimpanzee obligingly taps at the keyboard and after a period of time the writer is disappointed to find that the chimpanzee has only managed to turn out the worst of Shakespeare's tragedies! Such stories are amusing only to the extent that the audience has some realisation of how minute is the likelihood of the portrayed event. Yet, information produced by suitably random processes need not be meaningless strings of symbols. Random events can lead to the generation of meaningful information but such production is, in practice, extremely small in content and very rare. Of course, the more detailed such information is, the smaller is the probability of its generation by a random process. Although the random production of meaningful information would be surprising, upon confirming its random 
origin, one would have to accept the situation even though it would run counter to the standard conception of knowledge acquisition.

It should be clear now that time travel to the past (or any form of 'backwards' causation) is not necessary for the appearance of non-invented information (Riggs 1987, p.108; Smith 1997, p.371 n.11; Dainton 2010, p.138). Nevertheless, despite randomly produced information not being discovered or thought up by anyone, an origin can be specified for the creation of the information (i.e. a single point in space and time). The pertinent issue is that, even in normal circumstances, information can be generated but not invented or discovered. Therefore, any philosophical prejudice against the causal loop account based solely on the feature that information propagating around a causal loop may not be discovered or invented, is not justified. The essential difference between the causal loop version of knowledge acquisition and the randomly produced one is that no particular point in space and time or single event can be specified as the origin of the information 'circulating' the causal loop.

\section{WHAT'S MISSING?}

We have seen that origins in space and time are needed for the information carriers in the causal loop account (carriers, as there must be more than one) since these are entities that are governed by physical laws and processes. Given that non-invented information is simply accessible from the carriers, the production of such information should be little more surprising than the production of information by random processes. Travel to an earlier time would, however, increase the probability of some instances of noninvented information being produced above the probability of the same information arising randomly. The amount to 
which the probability of the non-invented information is raised will depend (to some extent) upon the method used to convey the information to the past. Even when we have granted that the loop process need not violate physical laws (including the Second Law of Thermodynamics) so that it is physically possible to gain knowledge by this method, knowledge acquisition via a causal loop still remains somewhat inscrutable (contrary to the view expressed in Meyer 2012). The persistence of the mystery arises from there being a piece missing from the causal loop explanation and we turn to this next.

The Temporal Epistemic Anomaly relies crucially on the existence of special information, the use of which would permit some form of complex message to be sent to an earlier time. We shall generally refer to this type of information (e.g. the design blueprints of a time travel machine) as 'time travel documentation' (TTD). Consider now what would be minimally required for TTD to be accessible from a causal loop:

1) The TTD is used by someone to make a means of transmitting information to the past (e.g. 'backwards'-in-time transmitter, time travel machine);

2) The content of the TTD is represented in a physical carrier that is suitable for transmission to the past (e.g. a 'backwards'-in-time modulated signal);

3) The physical carrier referred to in (2) is sent to the past;

4) On arrival in the past, the TTD is copied to a second physical carrier (e.g. a hardcopy is printed);

5) This second carrier later comes into the possession of the 'someone' referred to in (1). 
Although the scheme (1)-(5) looks sound enough (assuming 'backwards' causation is possible), it fails one basic test of validity. Since the use of TTD would allow for instances of non-invented information to be gained via some kind of time travel to the past, TTD is a logical precursor to the existence of any non-invented information accessed from a causal loop. In the case of TTD itself being sent to an earlier time (so that a time transfer mechanism's design information is not created), the TTD would have to be its own logical precursor as this is necessary for the existence of the TTD. It is self-evidently absurd for anything to be prior to itself in a logical sense (with perhaps the exception of logical tautologies which T'TD is not). The above scheme (1)-(5) is an example of what is called a pure fallacy (Barker 1974, p.192), with the fallacy arising from TTD being its own logical precursor. In light of this (invalid) requirement, we may infer that causal loops for content isolated T'TD are impossible. Therefore, an origin in space and time is needed for TTD but not necessarily for other kinds of information.

One might protest that this is all fine but the (alleged) self-defeating paradoxes of time travel are logically impossible too, yet one may still enquire into why selfdefeating causal loops do not occur when other (benign) causal loops are possible. What's different about the Temporal Epistemic Anomaly? The answer follows from scheme (1)-(5) being a pure fallacy and is more subtle. The case of TTD being sent to an earlier time is not a matter of how it is that a causal loop cannot transfer content isolated TTD completely around itself. A content isolated TTD loop cannot be instantiated at all as the necessary condition for its actuality (i.e. the use of existing TTD) does not obtain. The TTD referred to in (1) above cannot exist unless it was first produced at a specific point in space and time and only then might be used to affect the transfer of information to 
the past. This is the piece that has been missing from previous attempts at resolving the Anomaly.

In the context of relativistic physics, this result should be reflected in consistency conditions on spacetime models containing closed timelike curves (CTCs). Such consistency conditions have been discussed extensively in respect to the (alleged) paradoxes of time travel (e.g. Earman 1995; Earman et al. 2009; Smeenk \& Wüttrich 2011), as Douglas Kutach writes:

What appears to be a signature effect of CTCs is that some physical states that are possible according to the usual laws governing allowable local states cannot be extended via the usual laws of temporal evolution into a globally consistent solution. A restriction on the space of all physically possible fragments of history to those that can be extended by law into a globally consistent solution of a maximally large spacetime is called a consistency constraint (Kutach 2013, p.306, italics in original).

In a similar vein, TTD not being its own logical precursor could be given a mathematical expression in the form of a consistency constraint. What will constitute a suitable consistency constraint on spacetime models to achieve this will require formal investigation. 


\section{CONCLUDING REMARKS}

An origin in space and time is needed for all carriers of information as these are entities for which the usual physical limitations apply. Knowledge acquisition from a causal loop (with the exception of information that would allow some form of communication to an earlier time to be performed) is physically allowable, not logically inconsistent, and need not include the creation of the information content at any point in space and time. However, information that would allow its own transmission to the past is a special case where this type of transfer cannot even be attempted.

\section{REFERENCES}

AlBERT, D.Z. Time and Chance. Harvard: Harvard University Press, 2000.

AL-KHALILI, J. Black Holes, Wormboles \& Time Machines (2nd edn). London: Taylor and Francis, 2016.

BARKER, S.F. The Elements of Logic (2nd edn). New York: McGraw-Hill, 1974.

Berkovitz, J. "On Time, Causation and Explanation in the Causally Symmetric Bohmian Model of Quantum Mechanics". In Bouton, C. and Huneman, P. (eds), Nature of Time and the Time of Nature: Philosophical Perspectives of Time in Natural Sciences. Cham: Springer, 2017.

CAllender, C. "The Past Hypothesis Meets Gravity". In Ernst, G. and Hüttemann, A. (eds), Time, Chance, and Reduction: Philosophical Aspects of Statistical Mechanics. Cambridge: Cambridge University Press, 2010. 
Clegg, B. Build Your Own Time Machine: The Real Science of Time Travel. London: Duckworth Overlook, 2011.

Dainton, B. Time and Space (2nd edn). Durham, U.K.: Acumen, 2010.

Davies, P. How to Build a Time Machine. London: Penguin Books, 2001.

Deutsch, D. and LOCKWOOD, M. "The Quantum Physics of Time Travel". Scientific American (March issue), pp. 69-74, 1994.

Dugdale, J.S. Entropy and its Physical Meaning. London: Taylor and Francis, 1996.

EARMAN, J. Bangs, Crunches, Whimpers, and Shrieks: Singularities and Acausalities in Relativistic Spacetimes. Oxford: Oxford University Press, 1995. , SMEENK, C. and WÜTHRICH, C. "Do the Laws of Physics Forbid the Operation of Time Machines?". Synthese, 169, pp. 91-124, 2009.

FlORIDI, L. The Philosophy of Information. Oxford: Oxford University Press, 2011.

FrIEDMAN, J., MORRIS, M.S., NOVIKOV, I.D., ECheverria, F., Echeverria, G., ThOrNe, K.S. and Yurtsever, U. "Cauchy Problem in Spacetimes with Closed Timelike Curves". Physical Review D, 42, pp. 1915-1930, 1990.

Greene, B. The Fabric of the Cosmos: Space, Time and the Texture of Reality. London: Penguin Books, 2004.

GOTT III, J.R. "Closed Timelike Curves Produced by Pairs of Moving Cosmic Strings: Exact Solutions". Physical Review Letters, 66, pp. 1126-1129, 1991. 
Time Travel in Einstein's Universe: The Physical Possibilities of Travel Through Time. New York: Houghton Mifflin, 2001.

Hanley, R. "No End in Sight: Causal Loops in Philosophy, Physics and Fiction". Synthese, 141, pp. 123-152, 2004.

Horwich, P. Asymmetries in Time. Cambridge, MA: MIT Press, 1987.

ISMAEL, J. "Closed Causal Loops and the Bilking Argument”. Synthese, 136, pp. 305-320, 2003.

KAKU, M. Parallel Worlds: A Journey Through Creation, Higher Dimensions, and the Future of the Cosmos. New York: Doubleday Press, 2005.

Physics of the Impossible. London: Penguin Books, 2009.

KARnANI, M., PÄÄKKÖNEN, K. and AnNilA, A. "The Physical Character of Information". Proceedings of the Royal Society A: Mathematical, Physical \& Engineering Sciences, 465, pp. 2155-2175, 2009.

KuTACH, D. "Time Travel and Consistency Constraints". Philosophy of Science, 70, pp. 1098-1113, 2003.

"Time Travel and Time Machines". In A. Bardon and H. Dyke (eds), A Companion to the Philosophy of Time. Chichester: Wiley-Blackwell, 2013.

LANDAUER, R. "The Physical Nature of Information". Physics Letters A, 217, p. 188, 1996.

LEWIS, D. "The Paradoxes of Time Travel". American Philosophical Quarterly, 13, pp. 145-152, 1976.

LINDLEY, D. "Time Travel without Regrets". Physical Review Focus, 27 (5), 2011. Available at: http://physics.aps.org/story/v27/st5. 
Lobo, F. and Crawford, P. "Time, Closed Timelike Curves and Causality". In Buccheri, R., Saniga, M. and Stuckey, M. (eds), The Nature of Time: Geometry, Physics and Perception. Dordrecht: Kluwer Academic, 2003.

LOCKWOOD, M. The Labyrinth of Time: Introducing the Universe. Oxford: Oxford University Press, 2005.

LOMBARDI, O. "What is Information?". Foundations of Science, 91, pp. 105-134, 2004.

Lossev, A. and NOVIKOV, I.D. "The Jinn of the Time Machine: Non-trivial Self-Consistent Solutions". Classical and Quantum Gravity, 9, pp. 2309-2321, 1992.

MALLETT, R.L. "The Gravitational Field of a Circulating Light Beam". Foundations of Physics, 33, pp. 13071314, 2003.

and Henderson, B. Time Traveller: A Scientist's Personal Mission to Make Time Travel a Reality. London: Penguin Books, 2008.

Meyer, U. "Explaining Causal Loops". Analysis, 72, pp. 259-264, 2012.

Mingers, J. and STANDING, C. "What is information? Toward a theory of information as objective and veridical". Journal of Information Technology 33, pp. 85104, 2018.

Monton, B. "Time Travel Without Causal Loops". The Philosophical Quarterly, 59, pp. 54-67, 2009.

MOrRIS, R. Time's Arrows: Scientific Attitudes Towards Time. New York: Simon \& Schuster, 1985.

, Thorne, K.S. and YurTSEVER, U. "Wormholes, Time Machines, and the Weak Energy Condition". Physical Review Letters, 61, pp. 1446-1449, 1988. 
NAHIN, P. Time Macbine Tales: The Science Fiction Adventures and Philosopbical Puzzles of Time Travel. Cham: Springer International Publishing, 2017.

NikOLIĆ, H. "Causal Paradoxes: A Conflict Between Relativity and the Arrow of Time", Foundations of Physics Letters 19, pp. 259-267, 2006.

Patel, V.M. and LinewEAVER, C.H. "Solutions to the Cosmic Initial Entropy Problem without Equilibrium Initial Conditions", Entropy 19, pp. 4111-411-9, 2017.

Popovic, M. "The Second Law of Thermodynamics Forbids Time Travel". Cosmology, 18, pp. 212-222, 2014.

ReICHENBACH, H. The Direction of Time (ed. M. Reichenbach). Berkeley and Los Angeles: University of California Press, 1956.

REIF, F. Fundamentals of Thermal and Statistical Physics. Tokyo: McGraw-Hill- Kosahusha, 1981.

RigGS, P.J. "A Critique of Mellor's Argument Against 'Backwards' Causation". British Journal for the Philosophy of Science, 41, pp. 75-86, 1991.

"The Principal Paradox of Time Travel". Ratio, 10, pp. 48-64, 1997.

"The Physical State of the Universe in the Planck Era”. Zeitschrift für Naturforschung A: Journal of Physical Sciences, 73, pp. 533-537, 2018.

RALPH, T.C. and DOWNES, T.G. "Relativistic Quantum Information and Time Machines". Contemporary Physics, 53 (1), pp. 1-16, 2012.

SMEENK, C. and WÜTTRICH, C. "Time Travel and Time Machines". In C. Callender (ed.), The Oxford 
Handbook of Philosophy of Time. Oxford: Oxford University Press, 2011.

SMITH, N.J.J. "Bananas Enough for Time Travel?". British Journal for the Philosophy of Science, 48, pp. 363-389, 1997.

STOWE, K. An Introduction to Thermodynamics and Statistical Mechanics (2nd edn). Cambridge: Cambridge University Press, 2007.

TAYLOR, E.R. "The Thermodynamics of Time Travel", Universal Journal of Chemistry 3 (2), pp. 60-64, 2015.

ThORnE, K.S. Black Holes and Time Warps: Einstein's Outrageous Legacy. New York: W.W. Norton, 1994.

TIPPETT, B.K. and TSANG, D. "Traversable Acausal Retrograde Domains in Spacetime". Classical and Quantum Gravity, 34, pp. 095006-1-095006-12, 2017.

ToOmey, D. The New Time Travelers: A Journey to the Frontiers of Physics. New York: W.W. Norton, 2007.

VISSER, M. Lorentzian Wormholes: From Einstein to Hawking. New York: Springer, 1996.

Wasserman, R. The Paradoxes of Time Travel. Oxford: Oxford University Press, 2018.

WEINERT, F. The March of Time: Evolving Conceptions of Time in the Light of Scientific Discoveries. Berlin: Springer, 2013.

YuAN, X., ASSAD, S.M., ThOMPSON, J., HAW, J.Y., VEDRAL, V., RALPH, T.C., LAM, P.K., WEEDBROOK, C. and GU, M. "Replicating the benefits of Deutschian closed timelike curves without breaking causality". NPJ Quantum Information, 2015. Available at: http://www.nature.com/articles/npjqi20157 
ZEMANSKY, M.W. and DitTMAN, R.H. Heat and Thermodynamics. New York: McGraw-Hill, 1981.

ZINS, C. "Conceptual Approaches for Defining Data, Information, and Knowledge". Journal of the American Society for Information Science and Technology, 58, pp. 479-493, 2007.

(cc) EY

Manuscrito - Rev. Int. Fil. Campinas, v. 41, n. 3, pp. 01-28, Jul.-Sep. 2018. 


\title{
ERRATA
}

The article "THE TEMPORAL EPISTEMIC ANORMALY", with number of DOI: 10.1590/0100-6045.2018.V41N3.PR, published in volume MAN v41n3, p. 1:

Where it reads:

"THE TEMPORAL EPISTEMIC ANORMALY"

it should read:

“THE TEMPORAL EPISTEMIC ANOMALY"

Also in the header of the odd numbered pages where it reads:

\author{
"THE TEMPORAL EPISTEMIC ANORMALY" \\ it should read: \\ “THE TEMPORAL EPISTEMIC ANOMALY"
}

DOI: http://dx.doi.org/10.1590/0100-6045.2018.V41N3.ER

(cc) $\mathrm{BY}$

Manuscrito - Rev. Int. Fil. Campinas, Errata, 2018. 